\title{
LA INFLUENCIA DEL TAMAÑO INICIAL DEL POLVO DE ÓXIDO DE CIRCONIO EN FASE TETRAGONAL ESTABILIZADO CON ÓXIDO DE ITRIO EN LA FORMACIÓN DE UNA BARRERA DE PROTECCIÓN TÉRMICA
}

\author{
THE INFLUENCE OF THE INITIAL SIZE OF THE YTTRIUM OXIDE \\ STABILIZED TETRAGONAL PHASE ZIRCONIUM OXIDE POWDER ON \\ THE FORMATION OF A THERMAL PROTECTION BARRIER
}

\author{
Jhonattan Zavaleta' ${ }^{1}$, Olga Bondareva2(®) \\ IInstitute of Space Rocket Engineering, Samara National Research University, Samara, Rusia \\ ${ }^{2}$ Department of Metals Technology and Aviation Materials, Samara National Research University. Samara, Rusia
}

*E-mail: zavatisna@hotmail.es

Recibido (Recieved): 12/02/2019 | Aceptado (Accepted): 22/06/2020

\section{RESUMEN}

En este trabajo se estudian los efectos en las propiedades de porosidad y microdureza al usar distintos diámetros de polvos de óxido de circonio en fase tetragonal estabilizado con óxido de itrio (menores a $40 \mu \mathrm{m}$, entre 40 a $60 \mu \mathrm{m}$ y mayores a $80 \mu \mathrm{m}$ ) a depositar en la capa superior de un sistema TBC (barrera de protección térmica) de doble capa conformado por NiCoCrAlY y óxido circonio en fase tetragonal estabilizado con óxido de itrio usando el proceso de pulverización en plasma, con el propósito de encontrar el polvo adecuado y factible de usar como capa superior. Para ello se utilizaron instrumentos como el Microscopio electrónico de barrido TESCAN Vega SB con microanalizador dispersivo INCA Energy electronic microscope con el cual se pudo detectar la porosidad en distintas zonas de evaluación de la capa superior y el durómetro de Vickers para medir la microdureza. Comprobándose que con diámetros de partículas menores a $40 \mu \mathrm{m}$ las propiedades anteriormente mencionadas son las adecuadas para la conformación de la capa superior de óxido circonio en fase tetragonal estabilizado con oxido de itrio.

Palabras Claves: porosidad, microdureza, barreras de protección térmicas, proceso de pulverización en plasma, NiCrCoAlY y óxido zirconio en fase tetragonal estabilizado con oxido de itrio.

\section{ABSTRACT}

In this work we study the effects on the porosity and microhardness properties when using different tetragonal phase zirconium oxide stabilized with yttrium oxide as diameters powders (less than $40 \mu \mathrm{m}$, between 40 to $60 \mu \mathrm{m}$ and greater than $80 \mu \mathrm{m}$ ) to deposit in the upper layer of a TBC system (thermal barrier coating) of double layer formed by NiCoCrAlY and tetragonal phase zirconium oxide stabilized with yttrium oxide using the plasma spray process, in order to find the suitable powder and feasible to use as top layer. To this end, instruments such as TESCAN Vega SB scanning electron microscope with INCA Energy dispersive microanalyzer were used, with which it was possible to detect the porosity in different areas of evaluation of the upper layer and the Vickers durometer to measure the microhardness. Verifying that with particle diameters smaller than $40 \mu \mathrm{m}$, the aforementioned properties are adequate for the conformation of the tetragonal phase zirconium oxide stabilized with yttrium oxide such as top layer.

Keywords: porosity, microhardness, thermal protection barriers, plasma spraying process, NiCoCrAlY and zirconium oxide in tetragonal phase stabilized with yttrium oxide.

\section{INTRODUCCION}

Uno de los recubrimientos de alabes de turbina en la industria aeroespacial usado como capa superior de un sistema de barrera térmica (TBC) es el óxido de circonio en fase tetragonal estabilizado con óxido de itrio, ya que es considerado para aplicaciones de este tipo debido a su baja conductividad térmica, alto puntos de fusión y coeficiente de expansión térmica [ 1 , 2]. Los TBCs de zirconio-itria se consideran mejores a temperaturas de $1400{ }^{\circ} \mathrm{C}$ cuando se depositan sobre una capa de unión metálica [3,4] generalmente del tipo NiCoCrAlY, existen diversas formas de deposición de este recubrimiento, una de ellas es el proceso de pulverización en plasma [5], el cual consiste en utilizar una pistola de plasma en donde las partículas de polvo 
de este recubrimiento es fundido y depositado sobre la capa de unión metálica.

En este artículo se muestra la evaluación de la efectividad de este recubrimiento, para ello se realizó el análisis de la porosidad y microdureza; debido a que al aumentar la porosidad produce la reducción de la conductividad térmica; sin embargo, por encima de ciertos valores, este aumento puede degradar la integridad mecánica debido al aumento de la cohesión entre las láminas. Estas propiedades también se pueden controlar con mayor rigor si controlamos la geometría de los poros [6]. En el caso de la microdureza es común usar la prueba de Vickers, que es el método estándar para medir la dureza de los materiales [7].

\section{MÉTODOS Y MATERIALES}

Los materiales designados en este artículo son polvos de la aleación de NiCoCrAlY y óxido de circonio en fase tetragonal estabilizado con óxido de itrio, las composiciones de los polvos se pueden corroborar en la tabla 1, las cuales fueron realizadas con el Microscopio electrónico de barrido TESCAN Vega SB con microanalizador dispersivo INCA Energy.

Para empezar con el proceso de deposición sobre la superficie es necesario seguir el siguiente procedimiento: el cual consiste en un chorro de arena sobre superficies de la muestra, la cual es una superlación de níquel ZhS6F, con el objetivo de generar una rugosidad y un tipo de anclaje mecánico. Luego se procede a la aplicación de los siguientes materiales de revestimiento; la primera capa es un polvo de NiCoCrAlY,

TABLA I

Composición química que existe en el polvo NiCoCrAlY y del óxido de circonio en fase tetragonal estabilizado con óxido de itrio ( $\left(\mathrm{rO}_{2}+8 \%\right.$ \%2O3)

\begin{tabular}{|c|c|c|c|c|c|c|c|c|c|c|}
\hline \multirow[b]{2}{*}{ Sustancia } & \multicolumn{9}{|c|}{ Porcentaje en masa de los elementos (\%) } & \multirow[b]{2}{*}{ total } \\
\hline & $\mathrm{C}$ & $\mathrm{O}$ & Al & $\mathrm{Cr}$ & Co & $\mathrm{Ni}$ & $\mathrm{Y}$ & $\mathrm{Zr}$ & $\mathrm{Hf}$ & \\
\hline $\mathrm{NiCOCrALY}$ & 4.57 & 1.81 & 11.00 & 18.47 & 19.65 & 42.74 & 1.76 & - & - & 100 \\
\hline $\mathrm{ZrO}_{2}+8 \% \mathrm{Y}_{2} \mathrm{O}_{3}$ & 8.02 & 33.36 & 2.22 & - & - & - & 6.63 & 48.69 & 1.08 & 100 \\
\hline
\end{tabular}

la segunda capa es un polvo de óxido de circonio en fase tetragonal estabilizado con óxido de itrio de fase tetragonal. Para las muestras en donde se depositó los recubrimientos tienen formas de alabes de turbina a gas las cuales fueron dividas longitudinalmente en partes más pequeñas.

La deposición de las capas de recubrimiento se realizó con el proceso de pulverización en plasma los parámetros de la deposición fueron a presión ambiental (1 atm) y las temperaturas del plasma fue cercana a los $1000^{\circ} \mathrm{C}$, en el caso de la capa superior de óxido de circonio en fase tetragonal estabilizado con óxido de itrio se designaron tres tipos de diámetro de polvos: menores a $40 \mu \mathrm{m}$, entre 40 a $60 \mu \mathrm{m}$ y mayores a $80 \mu \mathrm{m}$; y para la capa intermedia de NiCoCrAlY se utilizaron polvos entre 22.23 y $33.39 \mu \mathrm{m}$ de diámetro. Estos fueron colocados en tres tipos de muestras distintas, divididas según los diámetros de polvo de óxido de circonio en fase tetragonal estabilizado con óxido de itrio.

En la detección de la porosidad se utilizó el Microscopio electrónico de barrido TESCAN Vega SB para visualizar y contabilizar los poros en la capa superior de óxido de circonio en fase tetragonal estabilizado con óxido de itrio.

En la detección de la microdureza Vickers, se utilizó la maquina llamada durómetro, la cual somete a la superficie de la muestra con una carga estándar de
$0.025 \mathrm{kgf}$ durante un tiempo de $10 \mathrm{~s}$ por medio de un diamante en forma de pirámide, la diagonal de la indentación resultante se mide bajo un microscopio electrónico, este proceso es repetido en un total de 11 pruebas para cada tipo de óxido de circonio en fase tetragonal estabilizado con óxido de itrio de distinto diámetro de polvo a depositar (menor que $40 \mu \mathrm{m}$, entre 40 a $60 \mu \mathrm{m}$ y mayor que $80 \mu \mathrm{m}$ ). Los datos obtenidos se sometieron a la siguiente fórmula [7]:

Donde:

$$
H V=1.854 \frac{F}{D^{2}} \ldots \text { (1) }
$$

F: carga aplicada, Kgf

D: longitud de la diagonal impresa, $\mathrm{mm}$.

\section{RESULTADOS Y DISCUSIONES}

\subsection{POROSIDAD}

La capa superior (óxido de circonio en fase tetragonal estabilizado con óxido de itrio) en los recubrimientos aplicados es la única que presenta porosidades, estas porosidades son visibles en mayor cantidad cuando el diámetro de polvos depositados de óxido de circonio en fase tetragonal estabilizado con óxido de itrio es menor (tabla 2); mientras que el diámetro de las porosidades aumenta cuando el diámetro de polvos depositados de óxido de circonio en fase tetragonal estabilizado con óxido de itrio es mayor (tabla 2); las razones de este incremento es 
debido al proceso de formación de la capa de superior, ya que en la pulverización con plasma, la microestructura de los recubrimientos depende en gran medida de las condiciones de procesamiento como el diámetro del polvo.

Los polvos depositados (splats) están separados por poros interlaminares resultantes de la rápida solidificación de las láminas, las cuales son muy finas formados por contacto incompleto entre los polvos depositados o alrededores de las partículas sin fundir, y grietas debido a tensiones térmicas y tensiones de relajación de enfriamiento. La presencia de grietas también aumenta la tolerancia a la deformación y mejora la resistencia al choque térmico para los TBC en servicio, estas son más visibles cuando los diámetros de polvo depositados es mayor a $80 \mu \mathrm{m}$ en comparación con los otros diámetros de polvos depositados (Figura 1), estas grietas se originan a partir de las tensiones térmicas que surgen del enfriamiento rápido durante el proceso de pulverización [7].

El porcentaje de porosidad es determinado en un área fotografiada por el microscopio (Tabla 2), los cuales son vacíos existente con respecto al área total, obteniendo una tendencia de aumento con respecto a los diámetros de polvo de óxido de circonio en fase tetragonal estabilizado con óxido de itrio depositado (Tabla 2 y Figura 1), la razón de esta tendencia es debido a que el área de los espacios vacíos de los poros dependen tanto directamente del diámetro de los mismos como de su distribución de estos en el área analizada (número de poros).

TABLA II

Valores estadísticos de los poros en la capa de óxido de circonio en fase tetragonal estabilizado con óxido de itrio ( $\mathrm{ZrO} 2+8 \%$ Y2O3) en función del tipo de diámetro de partícula depositada para generar esta capa.

\begin{tabular}{cccccccccc}
\hline & \multicolumn{3}{c}{ Diámetro de poro, $\mu \mathrm{m}$} & \multicolumn{3}{c}{ Número de poros } \\
\hline $\begin{array}{c}\text { Diámetro de polvo } \\
\left(\mathrm{ZrO}_{2}+8 \% \mathrm{Y}_{2} \mathrm{O}_{3}\right) \mu \mathrm{m}\end{array}$ & Min. & Max. & $\begin{array}{c}\sigma \\
\text { Des. }\end{array}$ & Promedio & Min & Max. & $\begin{array}{c}\sigma \\
\text { Des. }\end{array}$ & Promedio & Porosidad (\%) \\
$<40$ & 21.47 & 35.58 & 2.30 & 24.25 & 50 & 64 & 2.00 & 57.25 \\
$40-60$ & 7.72 & 64.63 & 3.45 & 31.71 & 45 & 59 & 1.89 & 51.2 & 14.51 \\
$>80$ & 20.31 & 86.2 & 4.32 & 45.35 & 45 & 32 & 1.75 & 38.6 & 31.48 \\
\hline
\end{tabular}

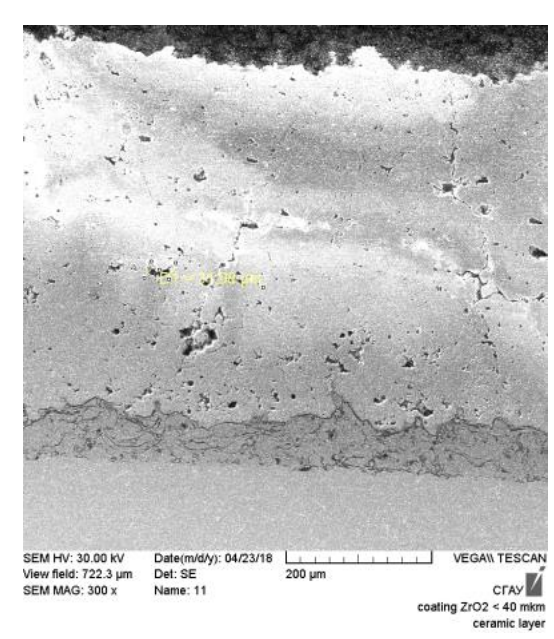

a.

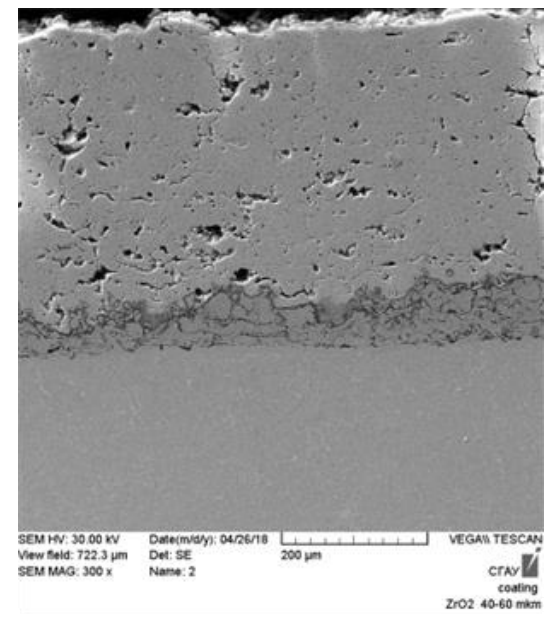

b.

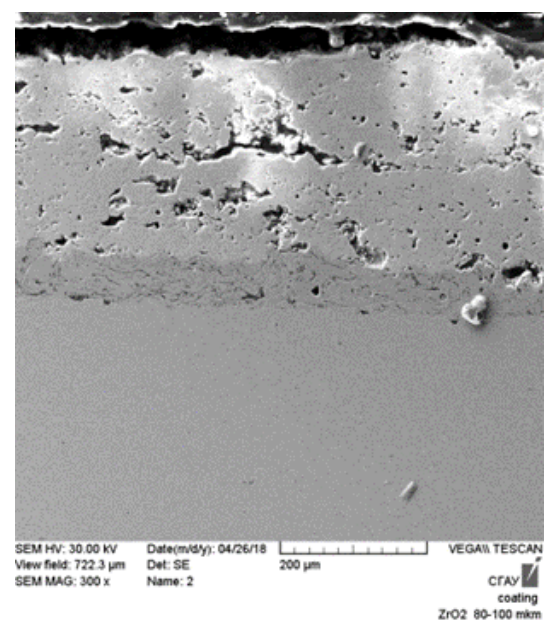

c.

Fig.1 El óxido de circonio en fase tetragonal estabilizado con óxido de itrio depositado en diferentes diámetros de polvo: a. Menores a 40 um, b. Entre 40 and $60 \mu \mathrm{m}$ y c. Mayores a $80 \mu \mathrm{m}$. 
TABLA III.

Valores estadísticos de las diagonales de la indentación y dureza Vickers (HV) en la capa de superior para distintos diámetros de polvo depositados

\begin{tabular}{cccc}
\hline & \multicolumn{3}{c}{$\begin{array}{c}\text { diámetro de Polvo depositado } \\
\text { de } \mathrm{ZrO2}+8 \% \mathrm{Y}_{2} \mathrm{O}(\mu \mathrm{m})\end{array}$} \\
\hline Parámetros & menor a 40 & entre 40-60 & mayor a 80 \\
D promedio, & 8.4 & 11.6 & 14.2 \\
D max, & 8.7 & 11.8 & 14.6 \\
D min, & 8.2 & 11.3 & 13.9 \\
O des, & 0.7 & 0.2 & 0.3 \\
HV & 802.1 & 350.8 & 231.1 \\
\hline
\end{tabular}

\subsection{DUREZA}

Este análisis involucra a la capa superior de óxido de circonio en fase tetragonal estabilizado con óxido de itrio, en ella se utilizó el instrumento durómetro para poder determinar el valor de dureza Vickers (HV), la zona donde se realizó la prueba tiene una forma rómbica y piramidal hundida debido a la fuerza aplicada por la indentación, además presenta dos diagonales (mínima y máxima), las cuales con ayuda de la ecuación 1 se designa los valores de dureza Vickers (HV) (Tabla III).

Los valores designados de microdureza para cada tipo de capa superior de óxido de circonio en fase tetragonal estabilizado con óxido de itrio (diámetro de polvo depositado) son los promedios y estos tienen una relación de decrecimiento a medida que aumenta el diámetro de partícula depositada (Tabla III), esto se debe al tipo de diámetros de polvo depositado, ya que son más sencillas de fundir cuando son pequeñas en el proceso de deposición, generando láminas más compactas uniéndose de mejor manera incrementando el anclaje entre ellas y por consecuente son más duros, además este tipo de cerámico ( $\mathrm{ZrO} 2)$ en su forma tetragonal la cual se utilizó en este experimento tiene un alto valor del módulo Young y de dureza; por la presencia de $\mathrm{Y}_{2} \mathrm{O}_{3}$ en su estructura dando esta estabilidad [8].

\section{CONCLUSIONES}

El polvo de óxido de circonio en fase tetragonal estabilizado con óxido de itrio con diámetro menor a $40 \mu \mathrm{m}$ utilizado como capa superior del sistema de recubrimiento TBC es el más factible en usar debido a su alta dureza y bajo porcentaje de porosidad.

La dureza de la capa superior de óxido de circonio en fase tetragonal estabilizado con óxido de itrio con diámetro de polvo menor a $40 \mu \mathrm{m}$, es mayor por presentar un menor número de grietas en la estructura.

\section{AGRADECIMIENTO}

Agradezco al Instituto de tecnología espacial y de cohetes de la Samara National Research UniversityRusia por el soporte académico. También en especial al departamento de Tecnología de metales y materiales de aviación por el soporte en la investigación científica, técnica y económica para llevar a cabo este trabajo.

\section{APENDICE}

Para determinar el porcentaje de porosidad en la capa superior entre los distintos tipos de diámetros de polvos depositados de $\mathrm{ZrO}_{2}+8 \% \mathrm{Y}_{2} \mathrm{O}_{3}$, se realiza midiendo el espesor de dicha capa usando el Microscopio electrónico de barrido TESCAN Vega SB con microanalizador dispersivo INCA Energy y el ancho de la imagen capturada en la zona medida, permitiendo obtener un área de forma rectangular promedio.

En el caso de diámetros de polvo depositado de $\mathrm{ZrO}_{2}+8 \% \mathrm{Y}_{2} \mathrm{O}_{3}$ menores que $40 \mu \mathrm{m}$ se calculó que el espesor de la capa superior es $422.71 \mu \mathrm{m}$ y el ancho de la imagen capturada por el microscopio es $731.6 \mu \mathrm{m}$ generando un área:

$$
\begin{gathered}
\text { Área } C P=\text { espesor } C P \text { x ancho } C P \\
=422.7 \mu m \times 731.6 \mu \mathrm{m} \\
=309254.6 \mu \mathrm{m}^{2} \ldots(A .1)
\end{gathered}
$$

El área que ocupan los poros, se realiza considerando que todos son circulares para ello en la zona evaluada se toma el promedio de los diámetros medidos por el microscopio y se contabiliza el total de estos en dicha área evaluada.

En el caso de diámetros de polvo depositado de $\mathrm{ZrO}_{2}+8 \% \mathrm{Y}_{2} \mathrm{O}_{3}$ menores que $40 \mu \mathrm{m}$ se calculó que el promedio de los diámetros de poros de 10 áreas evaluadas es $24.3 \mu \mathrm{m}$ y el promedio de poros contabilizados es 57.5 generando un área:

$$
\text { Área de poros }=\pi \cdot \frac{\bar{D}^{2}}{4} \cdot x
$$




$$
\begin{gathered}
=\pi \cdot \frac{24.3 \mu m^{2}}{4} \cdot 57.5 \\
=26557.1 \mu m^{2} \ldots(A .2)
\end{gathered}
$$

Obtenidos los valores A.1 y A.2 se podrá calcular el porcentaje de porosidad, el cual es una relación entre dichos valores:

$$
\begin{gathered}
\% \text { Porosidad }=\frac{\text { Área de Poros }}{\text { Área } C P} .100 \% \\
=\frac{26557.1 \mu m^{2}}{309254.6 \mu m^{2}} \cdot 100 \% \\
=8.57 \% \ldots(\text { A. } 3)
\end{gathered}
$$

Para el cálculo del porcentaje de porosidad de los otros diámetros de polvos depositados de $\mathrm{ZrO} 2+8 \%$ $\mathrm{Y}_{2} \mathrm{O}_{3}$, se repetirá el procedimiento usando como datos la tabla 2 y las relaciones A.1, A.2 y A.3.

\section{REFERENCIAS}

[1] V.I. Bogdanovich y M.G. Giorbelidze, "Enhancing Thermal barrier coatings performance through reinforcement of ceramic topcoat", IOP Conference Series: Materials Science and Engineering, vol.156, no. 1, pp. 1-7, nov. 2016. DOI: 10.1088/1757-899X/156/1/012016 [2] V.I. Bogdanovich y M.G. Giorbelidze , "Analysis of the ceramic layer microstructure influence on plasma spray thermal barrier coating performance", IOP Conference Series: Materials Science and Engineering, vol. 286, no.1, pp. 1-7, dic. 2017. DOI: 10.1088/1757899X/286/1/012008

[3] D.B. Zaytsev, I.A. Treninkov y A.A. Alexeyev , "Ultradisped plastic distribution in heat-resistant Nickel Alloys", Aviation materials and technologies, vol. 1, no. 34, pp. 49-55, 2015 DOI: 10.18577/2071-9140-20150-1-49-55 (en ruso).

[4] S.M. Meier y D.K. Gupta, "The evolution of thermal barrier coatings in gas turbine engine applications", Transactions of the ASME, vol. 116, no.1, pp. 250-257, ene. 1994. DOI: 10.1115/1.2906801

[5] V.I.Bogdanovich, S.B. Maryin, I.A. Dokukina y M.G. Giorbelidze, "Development of coatings composition and equipment for repair and strengthening of power generating unit elements by plasma spraying", Tsvetnye Metally, vol. 5, no. 1, pp. 56-62, jun. 2016. DOI: $10.17580 /$ tsm.2016.05.09

[6] V. Teixeira , "Numerical analysis of the influence of coating porosity and substrate elastic properties on the residual stresses in high temperature graded coatings", Surface and coating technology, vol. 146-147, pp. 79-84, oct. 2001. DOI: 10.1016/S0257-8972(01)014578

[7] V.C. Zolotoryevskij, "Mechanical properties of metals", Misis: Moskva, ed.3, pp. 272-273, 1998 (en Ruso).
[8] T.J. DeMasi-Marcin y D.K. Gupta, "Protective coating in the gas turbine engine", Surface and coating technology, vol. 68-69, pp. 1-9, dic. 1994. DOI: 10.1016/0257-8972(94)90129-5

Los artículos publicados por TECNIA pueden ser compartidos a través de la licencia Creative Commons: CC BY 4.0. Permisos lejos de este alcance pueden ser consultados a través del correo revistas@uni.edu.pe 